\title{
Dice Method: A Novel Approach to Evaluate Stress States Across a Retaining Wall Backfill
}

\author{
K. K. Pabodha M. Kannangara ${ }^{1}$, Shi-Yu Xu' \\ ${ }^{1}$ City University of Hong Kong \\ Kowloon, Hong Kong \\ kkannanga2-c@my.cityu.edu.hk \\ ${ }^{2}$ City University of Hong Kong \\ Kowloon, Hong Kong \\ shiyuxu@cityu.edu.hk
}

\begin{abstract}
An analytical approach to predict the stress distribution across a frictional-cohesive backfill, considering pseudo-static inertia forces, is presented herein. Vertical slices adopted in a recently proposed log-spiral-Rankine method are discretized further into dices. The governing equations to determine unknown inter-dice forces acting on a dice are formulated by considering the requirements of force equilibrium, balance of angular moment, and a shape function for the shear stress distribution. Local and global iteration schemes are employed to solve highly coupled non-linear equations. Outcome of the proposed method for the variations of the normal and shear stresses are shown sound agreement with the results obtained from finite element analysis. The information of this method can be used to analyse the strain distribution, and then the deformation of the backfill, which can create a basis to develop a performance based design criteria for earth retaining systems.
\end{abstract}

Keywords: Slice Method, Limit-Equilibrium, Earth Pressure, Log-Spiral-Rankine, Cohesion.

\section{Introduction}

Earth retaining systems are often found in cut and fill applications (e.g., the retaining walls use in building construction, etc). Conventionally, these systems are designed following the force-based approach by considering the lateral earth thrust and its point of application. However, more advanced approach such as the Performance Based Design (PBD) is already adopted in building codes [1], yet, implementation is limited in design of earth retaining systems due to lack of essential information such as uneven settlement to do loss estimation. Therefore, analysis of stress within the backfill is important as it can later be transformed to strain (and finally displacement).

Analysis of soil-structure interaction is a complex problem in geotechnical engineering. In practice, computer applications based on advanced numerical theories like Finite Element (FE) or full-scale test are available options to tackle the problem. Even though, the accuracy wise these approaches are highly rated, demand of experience, funds, space, and time have made those impractical to use in routine design work. Consequently, practitioners are acknowledged and comfortable with simple earth pressure analysis models albeit not perfect. Earth pressure analysis models can mainly be segregated into two groups under the limit analysis [2] - [3]. The models in the first group consider the stress solution approach (e.g., the Rankine model [4]) with no requirement of a presumed rupture surface. Second group consists with the models which employ the limit equilibrium and/or upper bound kinematical approach integrate with a yield criterion where information about stress distribution is absent. Moreover, realistic assumption of a failure surface geometry is a crucial factor for accuracy of solutions (especially under the passive case) obtained from the models in the second group. For example, well-known models such as the Coulomb [5] and the Mononobe-Okabe (MO) [6] - [7] are assumed simple planar failure surface. However, it has reported that the models assume planar failure surface (including both the Coulomb and the MO models) are often yield non-conservative solutions for the passive case especially when the roughness of the wall is high [8]. So, studies were extended to analyse the earth pressure by assuming more realistic non-linear shapes by incorporating techniques like the method of slices [9]. Nonetheless, slice methods are often required additional assumptions/simplifications as the problem is statically indeterminate in nature with the introduction of inter-slice forces. 
Recently, a limit-equilibrium method known as the log-spiral-Rankine (LSR) was introduced by considering a curvilinear failure surface, vertical method of slices, and major soil properties like soil friction and cohesion [3], [10] [12]. The method was originally proposed to compute static passive earth pressures and had been validated with several field test data [10] - [11], [13]. Later, it was extended by incorporating the pseudo-static inertia forces and compared against six prevailing earth pressure models with improved results [3]. $\mathrm{Xu}$ et al [12] reviewed the extended version and proposed an improved version (a.k.a. rigorous LSR method) with remedies to three drawbacks found. The LSR method is recommended for computation of seismic passive earth pressures [14]. Further, the simplified version of the rigorous LSR method is available as a simple closed-form equation too [15]. Currently, this method can capture the horizontal stress distribution along the failure surface, yet, the variation of the stresses along the vertical face of the slice is unknown due to limitations in vertical method of slices.

In summary, implementation of PBD requires additional information such as uneven settlement of the backfill to do loss analysis. The existing methods available to do so are either complex or time consuming (e.g., full-scale retaining wall test). Recently introduced LSR method has shown improved results in earth pressure analysis. However, this method is capable to compute the stress distribution in horizontal direction only. This study proposes a novel approach (dice method) to evaluate stress distribution across a backfill by further discretizing the slices utilized in the LSR method into dices. In following, the formulation of the dice method will be explained. The approach to solve the inter-dice normal and shear forces are elucidated. The validation of the dice method predications with 2D FE model is presented.

\section{Overview of the Dice Method}

Geometry of the dice method is bounded by an assumed composite (i.e., a log-spiral curve with a linear segment) failure surface, a vertical wall, and a levelled ground surface as shown in Fig. 1. The region above the linear segment is termed as the Rankine zone as the stress state in this region is equivalent to that of given by the Rankine theory. The logspiral geometry is governed by the locus 0 , the initial radius $\left(r_{o}\right)$, and the eccentric angle $\theta$ as annotated in Fig. 1, and can be established once the angle $\theta$ is known which is related to inclination angle at the Rankine zone $\left(\alpha_{R}\right)$ and the take-off angle at the bottom of wall $\left(\alpha_{w}\right)$ via log-spiral function. The geometry shown in Fig. 1 is formulated by following the procedure explained in the rigorous LSR method [12].

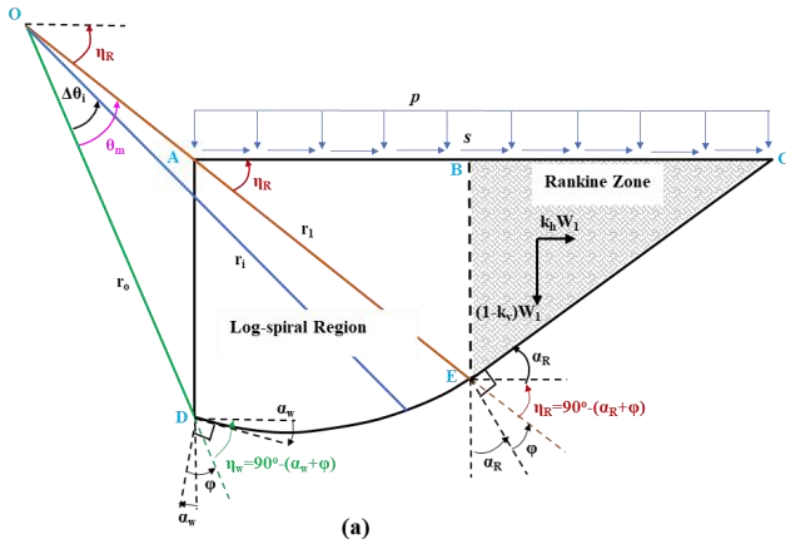

(a)

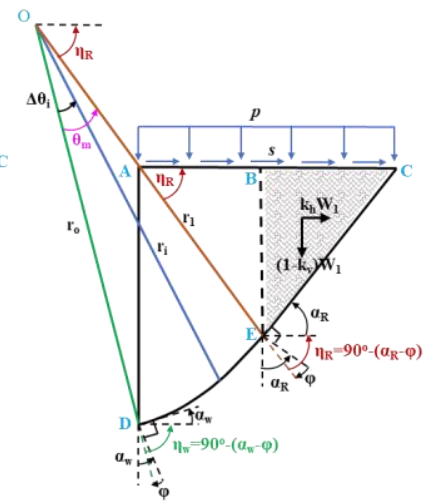

(b)

Fig. 1: Geometry of the mobilized soil body (a) under seismic passive case (b) under seismic active case.

Once the geometry of the soil body is established, the log-spiral region is discretized into dices and the inter-dice forces are introduced as shown in Fig. 2 (shown only for the passive case). For the active case, the directions of the interdice shear forces are interchanged, and provoked using term $j$ (i.e., $j=+1$ for passive case, and $j=-1$ for active case) when necessary. The Rankine zone is remained as a single slice and no further discretization is made as the shear stress of this region is solely induced by the body forces (i.e., including gravitational and inertia forces) acting on the soil body without any influence from the inter-slice forces [3]. 


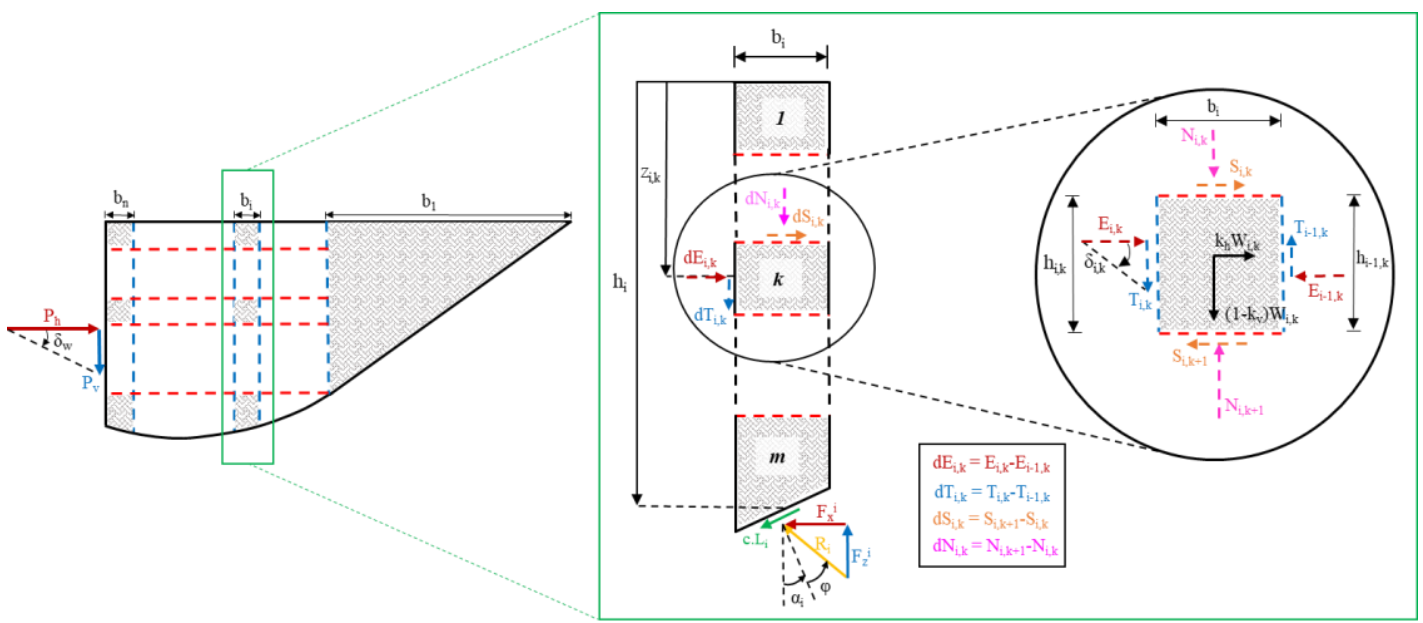

Fig. 2: Discretization of the mobilized soil body for seismic passive case.

Let an intermediate slice and a dice denote as $i$, and $k$, respectively. All the forces acting on a dice for seismic passive case can then be introduced in terms of indices $i$ and $k$ as graphically defined in Fig 2 (right). $E_{i, k}$ and $T_{i, k}$ are the normal and shear forces acting on the left face of the dice $k$ in slice $i ; E_{i-1, k}$ and $T_{i-1, k}$ are that of acting on the right face; $N_{i, k}$ and $S_{i, k}$ are the normal and shear forces acting on the top face of the dice $k$ in slice $i ; N_{i, k+1}$ and $S_{i, k+1}$ are that of acting on the bottom face. Further, all the incremental forces (e.g., $d E_{i, k}=E_{i, k}-E_{i-1, k}$, and so on) in each dice are defined in Fig. 2. Therefore, horizontal and vertical force equilibrium of a dice can be derived as in Eq. (1).

$$
\begin{gathered}
d E_{i, k}-d S_{i, k}=-k_{h} W_{i, k} \\
d T_{i, k}-d N_{i, k}=-\left(1-k_{v}\right) W_{i, k}
\end{gathered}
$$

where $W_{i, k}$ is the weight of the dice $k$ in slice $i$.

There are eight unknowns (i.e., a pair of normal and shear force acting on each face of a dice) for a typical dice as marked in Fig. 2 (right). However, the right face of the Rankine zone is reduced to a point (cf. Fig. 1), and the stress state of the left face is known (i.e., $\sigma_{z}=\left(1-k_{v}\right) \gamma z, \sigma_{x}=K \sigma_{z}$, and $\tau_{x z}=\left[k_{h} /\left(1-k_{v}\right)\right] \sigma_{z}$ where $K$ is the coefficient of lateral earth pressure). Also, the horizontal and vertical net forces (combination of cohesion and frictional forces) acting along the failure surface can be obtained by the rigorous LSR method [12] as in Eqs. (2a) and (2b), respectively.

$$
\begin{gathered}
F_{x, \text { net }}^{i}=F_{x}^{i}+c L_{i} \cos \alpha_{i}=\sigma_{x i} L_{i} \sin \alpha_{i}+\tau_{x z i} L_{i} \cos \alpha_{i} \\
F_{z, \text { net }}^{i}=F_{z}^{i}-c L_{i} \sin \alpha_{i}=\sigma_{z i} L_{i} \cos \alpha_{i}+\tau_{x z i} L_{i} \sin \alpha_{i}
\end{gathered}
$$

In other words, number of unknowns are reduced to 4 (i.e., $E_{i, k}, T_{i, k}, S_{i, k}$, and $N_{i, k}$ ) for a given dice as the inter-dice forces acting on the right $\left(E_{i-1, k}\right.$, and $\left.T_{i-1, k}\right)$ and the bottom $\left(N_{i, k+1}\right.$, and $\left.S_{i, k+1}\right)$ faces are always known either from the solution of the previous dice or the LSR method. Further, two more boundary conditions at the wall-soil interface (i.e., $P_{v}=P_{h}$ $\tan \delta_{w}$ ) as shown in Fig. 2 (left) and the surface tractions (i.e., $p$ and $s$ ) acting on the ground surface as shown in Fig. 1 must be satisfied. However, only two equations from the force equilibrium (refer Eq. (1)) are available to solve these four unknowns, therefore, two additional equations are required. These two equations are chosen by considering reasonable simplifications and approximations with a trial and error procedure. The first equation (Eq. (3a)) is essentially a simplification (i.e., $\tau_{x z-t o p}=\tau_{x z-\text { right }}$ ) based on the angular momentum in solid mechanics and the second equation (Eq. (3b)) is a shape function with a single unknown coefficient " $a$ " to model the shear stress distribution with depth (i.e., when $a=1$, it represents the Rankine shear stress distribution). These four equations (i.e., Eqs. (1) and (3)) are used in the dice method and the procedure to solve inter-dice forces are explained in the following section. 


$$
\begin{gathered}
S_{i, k} / b_{i}=T_{i-1, k} / h_{i-1, k} \\
\tau_{x z i, k}=\tau_{x z i}(1-b)\left(z_{i, k} / h_{i}\right)^{a}+\tau_{x z i} b
\end{gathered}
$$

where $b=\tau_{x z i, 1} / \tau_{x z i} ; \tau_{x z i, l}$ and $\tau_{x z i}$ are the shear stress at the top and bottom of slice $i$, respectively.

\subsection{Implementation of the Dice Method}

Implementation of the dice method is mainly considered in two cases, intermediate slice and the slice next to the wall. Each case, three zones (i.e., the bottom, an intermediate, and the top dices) are examined. In the dice method slice \#1 is considered as the Rankine zone. Also, for any typical dice (except the top dice denoted as 1 in Fig. 2), four governing equations are valid and can be derived as follows.

The shear force acting on the top face of a dice is found by rearranging the Eq. (3a), as in

$$
S_{i, k}=\left(T_{i-1, k} / h_{i-1, k}\right) b_{i}
$$

The horizontal normal force acting on the left face of a dice can be calculated by utilizing the relationships shown in Fig. 2 for incremental forces and Eq. (1a) as in Eq. (5).

$$
E_{i, k}=-k_{h} W_{i, k}+S_{i, k+1}-S_{i, k}+E_{i-1, k}
$$

If the surface tractions are assumed to be zero, coefficient $b$ of Eq. (3b) is zero and the shear force acting on the left face of a dice is given as in Eq. (6).

$$
T_{i, k}=\tau_{x z i}\left(z_{i, k} / h_{i}\right)^{a} h_{i, k}
$$

Similar to $E_{i, k}$, vertical normal force acting on the top face a dice is taken the form shown in Eq. (7) by incorporating the relationships for incremental forces given in Fig. 2 and the vertical force equilibrium equation (i.e., Eq. (1b)).

$$
N_{i, k}=F_{z, n e t}^{i}-\left(1-k_{v}\right) \sum_{e=k}^{m} W_{i, e}-\tau_{x z i} \sum_{e=k}^{m}\left(z_{i, e} / h_{i}\right)^{a} h_{i, e}+\sum_{e=k}^{m} T_{i-1, e}
$$

The dice method is always initiated from the bottom dice (denoted as $m$ in Fig. 2) of the first intermediate slice which is left to the Rankine zone. All four unknowns at the bottom dice $m$ of an intermediate slice $i$ can be found by replacing the index $k$ with $m$ in Eqs. (4) - (7). However, depending on the discretization, the right face of the dice $m$ can be reduced to a point as well (see Fig. 2). If that is the case, shear stress at the failure of slice $i$ is taken as the equivalent shear stress acting on the right face (i.e., $\left.T_{i-1, m} / h_{i-1, m}=\tau_{x z i}\right)$. Further, shear force acting on the bottom face of a dice $m\left(S_{i, m+1}\right)$ is essentially the corresponding horizontal net force (i.e., $\mathrm{F}_{\mathrm{x}, \text { net }}^{\mathrm{i}}$ ) given by Eqs. (2a).

The top dice of any slice in the dice method is indexed as 1 as mentioned before. Since no tractions are considered at the ground surface, the incremental normal and the shear forces acting on the top face are equal to the bottom forces (i.e., $d S_{i, 1}=S_{i, 2}$, and $d N_{i, 1}=N_{i, 2}$ ). Then, by integrating the incremental force relationships shown in Fig. 2 and Eq. (1), the horizontal normal and shear forces acting on the left face of the top dice can be yielded, as in

$$
\begin{gathered}
E_{i, 1}=-k_{h} W_{i, 1}+\left(T_{i-1,2} / h_{i-1,2}\right) \cdot b_{i}+E_{i-1,1} \\
T_{i, 1}=F_{z, n e t}^{i}-\left(1-k_{v}\right) \sum_{k=1}^{m} W_{i, k}-\tau_{x z i} \sum_{k=2}^{m}\left(z_{i, k} / h_{i}\right)^{a} h_{i, k}+\sum_{k=1}^{m} T_{i-1, k}
\end{gathered}
$$


Coefficient " $a$ " is still an unknown, and a residual equation is formed by replacing the index $k$ in Eq. (6) with 1 as shown in Eq. (10). The solutions for the coefficient " $a$ " for each slice are obtained by following the Newton's method.

$$
\gamma_{a}=\left.T_{i, 1}\right|_{E q .(9)}-\left.T_{i, 1}\right|_{E q .(6)}=F_{z, n e t}^{i}-\left(1-k_{v}\right) \sum_{k=1}^{m} W_{i, k}-\tau_{x z i} \sum_{k=1}^{m}\left(z_{i, k} / h_{i}\right)^{a} h_{i, k}+\sum_{k=1}^{m} T_{i-1, k}=0
$$

The special attention must be given to the slice next to the wall (identified as $i=n$ ) as the left face of the slice is in contact with the wall. Therefore, shear force acting on the left face of the dice in slice $n$ is governed by Eq. (11). In other words, Eq. (6) is no longer applied for this slice. Also, note that the shear force for the active case is considered by invoking the $j$ term as seen in Eq. (11).

$$
T_{n, k}=j E_{n, k} \tan \left(\delta_{w}\right)
$$

The remaining three unknowns (i.e., $S_{n, k}, E_{n, k}$, and $N_{n, k}$ ) for intermediate dice including bottom dice $m$ in this slice can be obtained by replacing index $i$ with $n$ in Eqs. (4), (5), and (7), respectively. However, in Eq. (7), the term relevant to the shear force acting on the left face must be replaced with Eq. (11). As for the top dice of slice $n$, two unknowns (i.e., $E_{n, 1}$ and $\left.T_{n, 1}\right)$ to be solved, yet, three equations are valid (i.e., Eqs. (1a), (1b), and (11)) which makes the problem overconstrained. So, Eq. (11) is dropped from implementation only at this case. The effect of this is found to be minimal as Eq. (11) is applied for all the other dices in slice $n$. Consequently, the two unknowns in the top dice are given by Eq. (8) and

(9) when the index $i$ is replaced with $n$. Note that the term related to the shear force acting on the left face of a dice in Eq.

(9) must be replaced with relationship given in Eq. (11).

\subsection{Evaluation of the Stress State along the Failure Surface}

The computation explained in section 2.1 to solve inter-dice forces are done in accordance to a stress state for an arbitrary geometry. When the geometry changes, stress state is changed as it is a function of inclination angle $\left(\alpha_{i}\right)$ as shown in Eq. (2). In the rigorous LSR method [12], stress state at the failure surface is solved using three residual equations as they assumed all the components (i.e., $\sigma_{z i}, \sigma_{x i}$, and $\tau_{x z i}$ ) of stress state are independent from each other. The first two residual equations derived in the rigorous LSR method (i.e., Eqs. (32) and (35) in [12]) remain unchanged. However, there is a slight change for the third equation (Eq. (38) in [12]) as in the dice method the variation of the inter-dice shear angle is allowed. Therefore, this equation is modified as the incremental inter-slice forces acting on the left face of a slice are equivalent to the summation of the incremental inter-dice forces acting on the left face of the dices (i.e., $d E_{i}=\sum_{k=1}^{m} d E_{i, k}$ and $\left.d T_{i}=\sum_{k=1}^{m} d T_{i, k}\right)$. Then, the final form is created by cancelling out the shear stress $\left(\tau_{x z i}\right)$ term in Eqs. (36) and (37) in [12]. These three highly coupled non-linear equations can be solved using Newton's method by following the procedure given in [12]. Once the stress state converges, the procedure stated in section 2.1 can be repeated. Ultimately, the lateral earth force can be calculated by integrating the horizontal normal forces along the height of the wall $\left(P_{h}=\sum_{k=1}^{m} E_{n, k}\right)$.

Additionally, $\mathrm{Xu}$ et al. [12] reported that the assumption of vertical normal stress as a linear function of depth (i.e., $\sigma_{z}$ $=\gamma \mathrm{z}$ ) can lead to inconsistent stress solution at the bottom of the wall. Therefore, a global iteration scheme was proposed to evaluate the normal vertical stress at the bottom of the wall. This step is unchanged in the dice method and the iterations are continued till the vertical normal stress at the bottom of the wall converges.

\section{Design of Soil Model for Numerical Verification}

The dice method is an analytical approach which enable to predict the stress distribution across a backfill. However, there is no proof found on an experimental study based on this objective for the best of authors knowledge, hence, computer application based on FE (ABAQUS) is chosen for verification purpose. Two ABAQUS 2D constitutive soil models are designed to validate the results obtained from the dice method. These models are done by complying with a real scale test on a seat-type abutment wall conducted under one-way cyclic loading at UCLA (hereafter known as UCLA test [16]). The tested wall was $1.67 \mathrm{~m}$ tall and $4.6 \mathrm{~m}$ wide which backfilled with silty sand. In fact, UCLA test was successfully validated by another FE model using PLAXIS [13]. Following the soil strength and elasticity parameters used 
by [13], the two constitutive soil models (i.e., lower bound (LB) and upper bound (UB)) are modelled for the dimensions shown in Fig. 3(a). The Drucker-Prager [17] plasticity model was used to model the non-linear behaviour of soil, and CPE6H (6-node quadratic with linear pressure) plain strain triangle elements [18] was used to mesh the soil body as illustrated in Fig. 3(a). Analysis was done in two steps where gravity load was applied under geostatic step and wall was displaced by $10 \mathrm{~cm}$ in the static general step. The wall-soil interaction was modelled by invoking the penalty method under tangential behaviour with a wall's frictional angle $\left(\delta_{w}\right)$ of $20^{\circ}$ as recommended by [16]. The force displacement curve for FE analysis, UCLA test data, and the dice method solutions for both LB and UB cases are plotted in Fig. 3(b). It can be see that the total horizontal force yielded from dice method are well agree with the FE analysis.

(a)

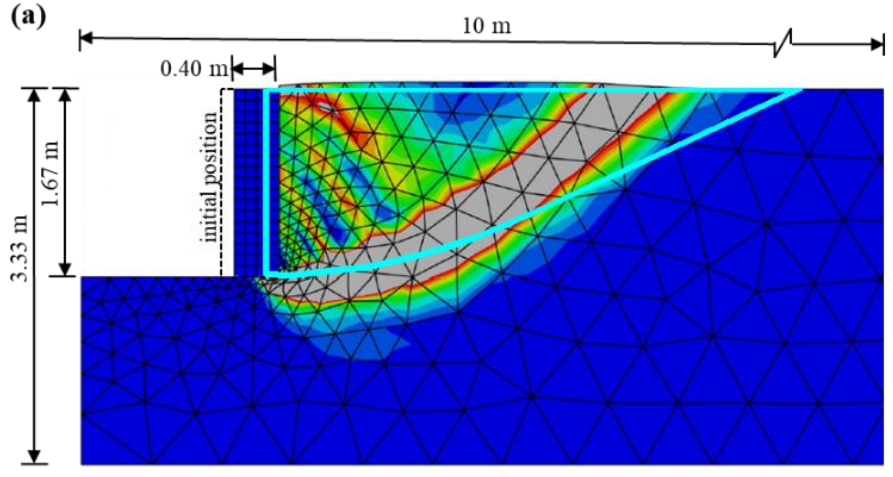

(b)

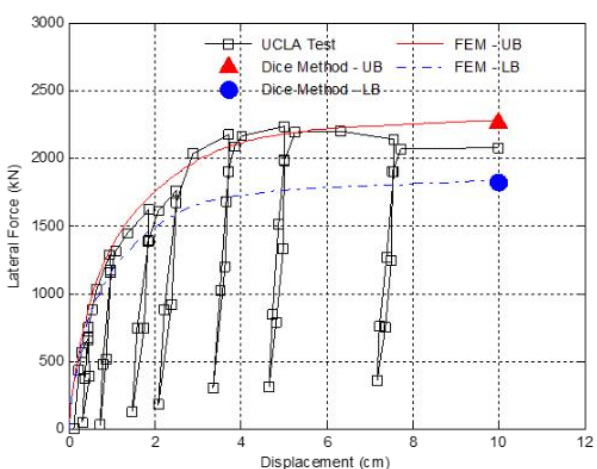

Fig. 3: Comparison of the dice method results with FE analysis (a) failure surface; (b) force-displacement relationship.

\section{Results and Discussion}

In the dice method, the corresponding stresses are calculated by dividing the inter-dice forces by the area of the face and assumed to be acting on the midpoint of the left face of a dice. For brevity, the comparisons of the dice method result with FE analysis are presented only for the LB case as UB case also shown similar agreement. Further, the FE results are selected up to the failure depth as the dice method unable to predict the solutions beyond that. The mobilized failure geometry obtained from the FE analysis and the dice method for LB case is shown in Fig. 3(a). It can be seen from Fig. 3(a) that the shape of the failure surface generated by the dice method is reasonably agreed with the FE results although the horizontal failure distance is overpredicted. Also, the mobilized soil body discretized into dices for LB case is shown in Fig. 4(a). The dice method outcomes for stress distribution shown in Fig. 4 are chosen from the slice $n$ (i.e., slice next to the wall), slice 4, and slice 1 (i.e., the Rankine zone) as marked in Fig. 4(a).

Variation of the vertical normal stress with depth yielded from the dice method and FE analysis are plotted in Fig. 4(b) for LB case. The filled markers represent the average vertical normal stresses which are generated from the dice method. In contrast, unfilled markers represent the vertical normal stresses which are obtained by the FE analysis at the centroid of relevant elements. The comparison in Fig. 4(b) shows that the dice method can capture the trend of $\sigma_{z}$ variation along the back of the wall reasonably well except at the bottom of the wall. The main reason behind the predictions to be overpredicted at the bottom could be the stress concentration phenomenon explained in the rigorous LSR approach [12]. They further explained that this is caused because of the $\sigma_{z}$ at the bottom of the wall is inversely proportional to the width of the slice next to the wall $\left(b_{n}\right)$. Consequently, if the mesh is finer, the predicted value for $\sigma_{z}$ at the bottom of the wall will be larger. In general, variation of the $\sigma_{z}$ predicted by the dice method is given an upper bound envelope for the FE results behind the wall. On the other hand, the dice method solution for the variation of $\sigma_{z}$ at the intermediate slice (e.g., slice 4) are underestimated the FE results. This could again be an effect of the aforesaid stress concentration phenomena. However, the dice method predictions are agreed well with FE solution for the variation of $\sigma_{z}$ at the Rankine zone.

The variation of the horizontal normal stress $\left(\sigma_{x}\right)$ with depth for the solutions obtained from the dice method and FE analysis are plotted in Fig. 4(c) for LB case. The data points are plotted by following the same pattern as $\sigma_{z}$ case. According to Fig. 4(c), dice method predication for the variation of the $\sigma_{x}$ is closely matched with FE solution for the most part of the wall height. However, like $\sigma_{z}$ case, estimation of the dice method at the bottom is much greater when compared to that of FE results. Further, FE results shows that the $\sigma_{x}$ is gradually dissipated when moves away from the wall. The dice 
method, however, is unable to capture this trend well, and overestimate the mobilized failure envelope as shown in Fig. 3(a).

Fig. 4(d) shows the variation of the shear stress $\left(\tau_{x z}\right)$ with depth for the dice method and FE analysis for LB case. The results show that the dice method predictions near the wall for $\tau_{x z}$ are well matched with FE analysis except the region close to the bottom of the wall. The predictions of the dice method for $\tau_{x z}$ at intermediate case (e.g. slice 4) are underpredicted the FE results like $\sigma_{z}$ case. Since this analysis is done for the static case, zero shear stresses are calculated from the dice method in the Rankine zone. FE solutions for the Rankine zone are also found to be very small as shown in Fig. 4(d). Shear stress distributions further emphasis that the variation of the inter-dice shear angle is non-linear along the depth for the log-spiral region (except the slice next to the wall).

(a)

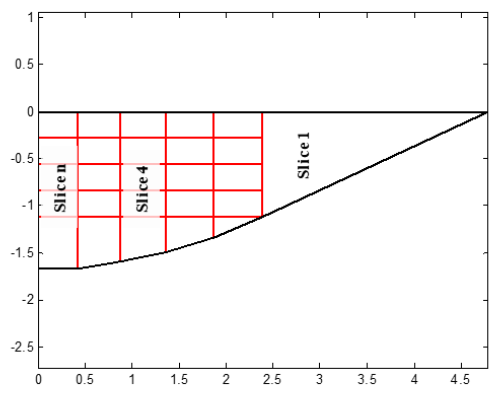

(c)

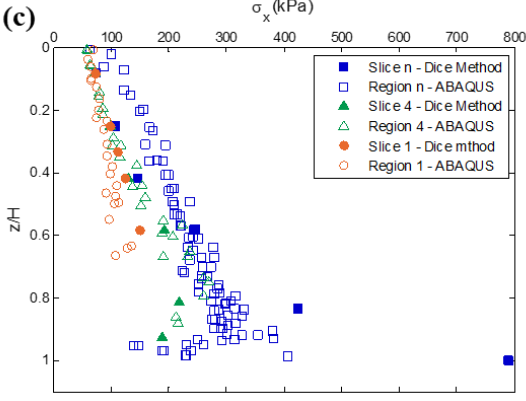

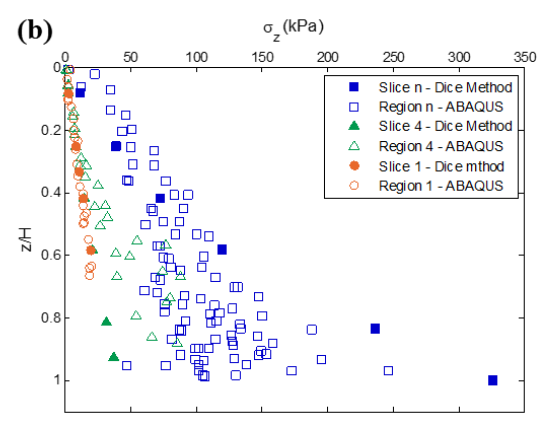

(d) $\tau_{x z}(\mathrm{kPa})$

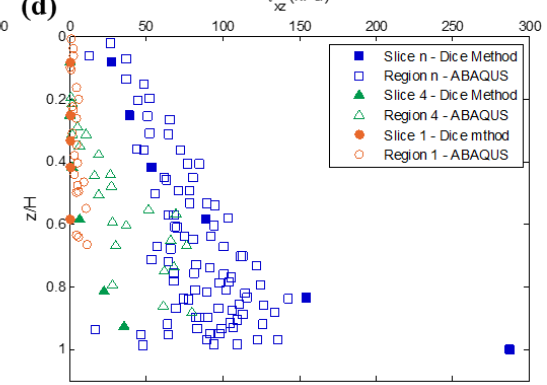

Fig. 4: (a) Discretized mobilized soil body in the dice method; comparison of the dice method and FE analysis solutions for the distribution of stress with the depth (b) vertical normal stress; (c) horizontal normal stress; (d) shear stress for LB case.

\section{Conclusion}

An analytical approach termed as the dice method is formulated to predict the variation of the stress state across the backfill. Keeping the recently developed LSR method as the base, the log-spiral region in the dice method is discretized into finite number of dices. Unknown inter-dice forces are solved by integrating the relationships obtained from the force equilibrium, balance of angular moment and a shape function for the shear stress distribution.

The dice method outputs are verified against the FE simulation which is modelled by complying with the parameters obtained from the full-scale abutment test done at UCLA. The comparison shows that the variation of the normal and the shear stresses along the back of the wall yielded from the dice method are well agree with the FE results for the most part of the wall height. However, dice method overpredicts the stress concentration phenomenon occurs at the bottom of the wall. The horizontal failure distance predicted by the dice method is overestimated FE solution as a result of underpredicting the reduction of the horizontal normal stress away from the wall. Further, it is observed that the inter-dice shear angle is a non-linear function of the depth within the log-spiral region. Currently, analysis of this method is limited to vertical wall with levelled backfill scenarios.

The stress distribution obtained from the dice method can be used to yield the strain distribution across the backfill. Once the strain distribution is known, the displacement of the wall and the uneven settlement can be found without a need of a complex approach like FE. Consequently, it enables to adopt PBD criteria for design of retaining wall systems. 


\section{References}

[1] Seismic Evaluation and Retrofit of Concrete Building (ATC-40). Applied Technology Council, Redwood City, California, 1996.

[2] W. F. Chen and X. L. Liu, Limit analysis in soil mechanics. Elsevier, Amsterdam, 1990.

[3] A. Shamsabadi, S-Y. Xu and E. Taciroglu, "A generalized log-spiral-Rankine limit equilibrium model for seismic earth pressure analysis," Soil Dynamics and Earthquake Engineering, vol. 49, pp. 197-209, 2013.

[4] W. J. M. Rankine, "On the stability of loose earth," Philosophical Transactions of the Royal Society of London, vol. 147, no. 1, pp. 9-27, 1857.

[5] C. A. Coulomb, "Essai sur une application des regles des maximis et minimis a quelques problemes de statique relatifs a l'architecture," Memoires de l'Academie Royale pres Divers Savants, vol. 7, 1776.

[6] N. Mononobe and H. Matsuo, "On the determination of earth pressures during earthquakes," Proc. World Engineering Conf, pp. 179-187,1929.

[7] S. Okabe, "General theory of earth pressure," J. Japan. Soc. of Civ. Eng., vol. 12, no. 1, pp. 123-134, 1926.

[8] G. Mylonakis, P. Kloukinas and V. Papantonopoulos, "An alternative to the Mononobe-Okabe equations for seismic earth pressures," Soil Dynamics and Earthquake Engineering, vol. 27, no. 10, pp. 957-969, 2007.

[9] D.H. Shields and A. Z. Tolunay, "Passive pressure coefficients by method of slices," J. of the Soil Mechanics and Foundations Division, vol. 99, no. 12, pp. 1043-1053, 1973.

[10] A. Shamsabadi, M. Ashour and G. Norris, "Bridge abutment nonlinear force-displacement-capacity prediction for seismic design," J. Geotech. Geoenviron. Eng., vol. 131, no. 2, pp. 151-161, 2005.

[11] A. Shamsabadi, K. M. Rollins and M. Kapuskar, "Nonlinear soil-abutment-bridge structure interaction for seismic performance-based design," J. Geotech. Geoenviron. Eng., vol. 133, no. 6, pp. 707-720, 2007.

[12] S-Y. Xu, A. Shamsabadi and E. Taciroglu, "Evaluation of active and passive seismic earth pressures considering internal friction and cohesion," Soil Dynamics and Earthquake Engineering, vol. 70, pp. 30-47, 2015.

[13] A. Shamsabadi, P. Khalili-Tehrani, J. P. Stewart and E. Taciroglu, "Validated simulation models for lateral response of bridge abutments with typical backfills," J. of Bridge Eng., vol. 15, no. 3, pp. 302-311, 2010.

[14] American Association of State Highway and Transportation Officials, AASHTO LRFD bridge design specifications. Washington, D.C., 2012.

[15] S-Y. Xu and K. K. P. M. Kannangara, "Semi-Analytical Approach to Evaluate Seismic Passive Earth Pressures Considering the Effects of Soil Cohesion and a Curvilinear Failure Surface," J. of Geotech. and Geoenv. Eng., vol. 143, no. 9, pp. 06017011, 2017.

[16] J. P. Stewart, E. Taciroglu, J. W. Wallace, E. R. Ahlberg, A. Lemnitzer, C. S. Rha, P. Khalili-Tehrani, S. Keowen, R. L. Nigbor and A. Salamanca, "Full scale cyclic testing of foundation support systems for highway bridges. Part II: Abutment backwalls," Structural and Geotechnical Engineering Laboratory, University of California, Los Angeles, Rep. UCLA-SGEL 2007/02, 2007.

[17] D. C. Drucker and W. Prager, "Soil mechanics and plastic analysis for limit design," Quarterly of Applied Mathematics, vol. 10, no. 2, pp. 157-165, 1952.

[18] ABAQUS, Version 6.7 Documentation, Simulia, Dassault Systèmes. RI, USA, 2007. 